\title{
Psicoanálisis: cambiar para permanecer ${ }^{1}$
}

\author{
José Jiménez Avello² \\ Madrid, España
}

\begin{abstract}
El psicoanálisis, por su propio desarrollo a lo largo de más de un siglo -si tomamos como fecha inicial 1900 tras la publicación de La Interpretación de los sueños-, está introduciendo cambios, y precisa introducir otros, respecto a sus concepciones iniciales. Lo que afecta tanto a cuestiones de orden teórico como práctico y técnico. Aquí se revisan sobre todo la noción de pulsión de muerte, y el par represión / clivaje en lo que hace a lo teórico, y sobre lo práctico los que fueron clásicos principios sobre la neutralidad del analista y la abstinencia en la cura, así como la preponderancia que se le concedió a la interpretación como factor terapéutico y el relieve que ha adquirido el manejo de la contratransferencia. Hay además reflexiones sobre los criterios de formación y validación de nuevos psicoanalistas.
\end{abstract}

Palabras clave: psicoterapia psicoanalítica, pulsión de muerte, clivaje, neutralidad, interpretación, contratransferencia, factores terapéuticos, formación.

Psychoanalysis, by its own development for more than a century - if we take 1900 as the initial date after the publication of The Interpretation of Dreams - is introducing changes, and needs to introduce others, with respect to their initial conceptions. This affects both theoretical, practical and technical questions. Here we review mainly the notion of the death instinct and the repression / cleavage pair in what makes the theoretical, and on the practical those that were classic principles on the neutrality of the analyst and abstinence in the cure, as well as the preponderance which was granted to interpretation as a therapeutic factor and the relevance that has acquired the handling of countertransference. There are also reflections on the criteria for training and validation of new psychoanalysts.

Key Words: Psychoanalytic psychotherapy, death instinct, cleavage, neutrality, interpretation, countertransference, therapeutic factors, training.

English Title: Psychoanalysis: change to stay

\section{Cita bibliográfica / Reference citation:}

Jiménez Avello, J. (2017). Psicoanálisis: Cambiar para permanecer. Clínica e Investigación Relacional, 11 (2): 324-336. [ISSN 1988-2939] [Recuperado de www.ceir.info ] DOI:

10.21110/19882939.2017.110207

\footnotetext{
${ }^{1}$ Texto de la conferencia dada en Acippia, Madrid el 27 de Enero de 2016

2 José Jiménez Avello es Médico, Psiquiatra y Psicoanalista. C./ San Bernardo, $643^{\circ}-11$ (entrada por C./ San Vicente Ferrer, 60) 28015 Madrid. E-Mail: jimenez.avello.psi@gmail.com
} 
Cuando se me propuso dar esta conferencia dejándome elegir tema, no lo dudé, porque el asunto del cambio en psicoanálisis lleva un tiempo rondándome. Comento que, como alternativa al título con que finalmente se ha convocado la charla, había propuesto otro, que no convenció a la autoridad competente, y que además me creaba un problema, porque era "Psicoanálisis: hacia un nuevo paradigma", y dudaba si escribirlo así, como una afirmación, o entre interrogaciones: "Psicoanálisis: ¿hacia un nuevo paradigma?". Necesitaría encontrar, para que respondiera a mi punto de vista, una forma de escribirlo entre la interrogación y la afirmación. Y no se me ocurre que hay entre medias.

En cualquier caso, lo que me pregunto es como es y será el psicoanálisis en el siglo XXI. Simplificando: ¿qué hay que añadir o profundizar para que siga vivo?, ¿qué hay que eliminar para evitar que pase al baúl de los recuerdos?.

Aunque hay que reconocer, que salvo para todo aquello que tenga que ver con la medición del tiempo, por lo demás, el cambio de siglo es simplemente el paso de un segundo al siguiente en el que cambia el día, el mes, el año, el siglo (y en nuestro caso incluso el milenio). Para nosotros (y para mi) hablar de un psicoanálisis del siglo XX y otro del siglo XXI no deja de ser sino un artificio, así que, en realidad de lo que voy a hablar es de evolución del psicoanálisis, aunque use esta muleta de comparar los siglos XX y XXI.

Una última advertencia antes de entrar directamente en materia. Voy a hablar del psicoanálisis, digamos "por dentro", y no de ningún psicoanálisis-fusión, que no practico, por lo que hablar de esas técnicas mixtas sería una impostura por mi parte. Las únicas "fusiones" que intento hacer en mi práctica son con el sentido común (del que se dice que es "el menos común de los sentidos"), y hago fusión también con el compromiso de trabajar a favor de la salud mental.

Y con éste último comentario, he empezado ya a entrar en materia.

El psicoanálisis que puede sobrevivir es el de intención terapéutica. Lacan decía que en psicoanálisis "la curación viene por añadidura" (Lacan (1983 [1954-55]), pero siempre me pregunto al leer esta frase, ¿por añadidura de que? La respuesta para algunos es que el psicoanálisis es una filosofía, o también, una teoría general del psiquismo humano. De ambas cosas hay que irse olvidando: como filosofía, el psicoanálisis es poco, y como teoría general del psiquismo, digo yo que algo tendrán que decir al respecto cognitivistas, neurobiólogos, humanistas, psiquiatras, sociólogos... y seguro que otros que no me vienen a la cabeza en este momento. Bajemos un poco entonces los humos y aceptemos un rol por un lado más humilde, pero por otro de gran calado. El psicoanálisis es un método expresa. Este material es para uso científico y profesional exclusivamente y puede contener información clínica sensible. Los editores no se responsabilizan de los contenidos de los autores. Dirigir las consultas sobre derechos y autorizaciones a ceir@psicoterapiarelacional.es 
terapéutico, hasta el punto de que, para mí en la actualidad, hablar de psicoanálisis o de psicoterapia psicoanalítica son dos formas de nombrar lo mismo.

Voy a tomar ahora ciertas nociones teóricas que no se si han cambiado o no, pero que en todo caso creo que deberían cambiar.

Empiezo por una noción, "altamente especulativa" según su autor, que creo habría que desterrar: la de pulsión de muerte, al menos tal y como la presenta Freud (Freud 1920) seguido por Melanie Klein. Es una noción introducida por Freud en un momento complicado de su vida, en 1920, --después de la guerra del 14 en la que ha llegado a tener a tres hijos movilizados, --aún persiste la penuria, la escasez y el hambre en Viena, --el año anterior (1919) ha muerto su gran amigo Anton von Freund y su discípulo Viktor Tausk se ha suicidado --y sobre todo, nada más empezar este año 20, ha muerto en pocos días de enfermedad su hija Sofía de la mal Ilamada "gripe española". Esto en lo íntimo de Freud, pero además en el campo psicoanalítico se ha pasado del optimismo terapéutico en los primerísimos tiempos, al pesimismo terapéutico cuando se ve que no todo el mundo mejora con el análisis, e incluso que algunos empeoran. Freud entonces, ante tan negro panorama, apela a una entidad desde el pesimismo, pero que además tal vez le sirva para encubrir los fracasos: se trata de la noción de pulsión de muerte que, por un lado (dice) es biológica, y por otro una especulación. "de altos vuelos", de nuevo según sus propias palabras.

Ferenczi tiene una pequeña nota en la que se opone radicalmente a Freud diciendo: "Nada más que pulsión de vida, la pulsión de muerte es un error (pesimista)", y más radical es aún Winnicott quien por carta escribe a Money-Kyrle (Winnicott 1989, "siento que Melanie haya hecho un esfuerzo tan grande para conciliar su punto de vista con la teoría de pulsiones de vida y de muerte, que a mi juicio es la mayor metedura de pata de Freud" (27.11.52). Coincido con ambos.

Los únicos autores que retomaron literalmente esta teoría pulsional freudiana fueron de hecho Melanie Klein y sus seguidores (tal vez por razones "diplomáticas"). Por lo demás, resulta revelador que autores que han retomado el asunto, en realidad hacen un uso muy distinto de la noción, introduciendo formas de entenderla que a mi entender serían igualmente interesantes, aunque dejaran de nombrarlo como pulsión de muerte (Green, Laplanche, Piera Aulagnier, Beno Rosemberg, Lacan...).

Termino este asunto con un posicionamiento aún más radical que en el que comenzaba hablar de la p.m. Nadie ha podido demostrar que exista tal entidad, ni biólogos ni trabajadores clínicos, pero si alguna vez se demostrara que existe, no dejaría por ello de ser expresa. Este material es para uso científico y profesional exclusivamente y puede contener información clínica sensible. Los editores no se responsabilizan de los contenidos de los autores. Dirigir las consultas sobre derechos y autorizaciones a ceir@psicoterapiarelacional.es 
descartable en análisis donde se convierte en justificación de los errores y fracasos del psicoanalista.

Otra cuestión de gran calado teórico es la progresiva conceptualización de un inconsciente distinto al reprimido, el único existente para Freud salvo por alguna mención de última hora, por lo demás confusa.

Él había escrito en 1899: "Aun en los sueños mejor interpretados es preciso a menudo dejar un lugar en sombras... ese es el ombligo del sueño, el lugar en que él se asienta en lo desconocido" (1900a). Es un pasaje de la Traumdeutung citado frecuentemente y de una calidad literaria y evocativa indudable.

Se entiende que es una metáfora, pero continuándolo como metáfora, y hasta cierto punto literalmente, además, el ombligo no nos une a lo desconocido, sino que es el resto de lo que nos unió a nuestra madre en una simbiosis de tipo parasitario. Es el resto, metafórico y (anatómico) que da cuenta del primer clivaje por traumatismo: el que separó al parásito niño del huésped madre. Es el momento que podríamos llamar de "clivaje primordial".

Clivaje, escisión vertical, fragmentación, etc., aunque con distintos matices, aluden a lo mismo. A la ruptura de la identidad integrada del sujeto. Desde ahora debo decir (o recordar) que el clivaje es la defensa característica ante situaciones traumáticas evitables o inevitables, siendo el modelo de los inevitables el originado en el nacimiento.

El enfrentamiento obsesivo de Freud (1926d) con Rank (1977 [1924]) y viceversa, en relación a la importancia del traumatismo del nacimiento, hay que situarlo en un plano no concebido en aquellos momentos, en el de la aparición primera de lo escindido, que ha de tener por tanto su huella en el psiquismo. Si bien Freud no conceptualizó hasta muy tarde en su obra, el "sorprendente" (sic, 1940e) mecanismo de la escisión vertical o clivaje, se le puede intuir que late en él desde su arcaico "Proyecto de psicología" (1950 [1895]). Aquí aparece la referencia a la "hilflosigkeit", la "helplessness", el "desamparo básico" en que se encuentra el recién nacido; habría que añadir: frente al amparo absoluto (parasitario) del que había gozado hasta entonces. Desamparo que proviene de la pérdida de la unidad madre-feto, clivada con el nacimiento.

El antídoto, nunca perfecto, a esta situación de desamparo, Freud lo denomina "experiencia primordial de satisfacción" del lactante, dicho a su manera. Digo a su manera, porque más bien cabría hablar de una "relación primordial de satisfacción", esa que mediante luz tenue, ambiente cálido, proximidad al cuerpo materno, suave canturreo, amamantamiento (a esto último es a lo que se refiere Freud como "experiencia primordial de satisfacción") trata de restaurar en la medida de lo posible aquel paraíso perdido del feto al convertirse en neonato. 
Muy importante para Rank que consideraba la angustia neonatal como matriz de toda angustia posterior, Freud disentía de Rank profusa y confusamente, puesto que la angustia de castración, su gran hallazgo, siguiendo a Rank quedaba destronada, y pasaba a ser un posición más, una metonimia más, de la angustia del nacimiento.

Pero lo que ni en uno ni en otro, ni en Freud ni en Rank, se plantea claramente es que, la travesía del canal del parto, el desplegamiento del árbol bronquial, la aparición del Ilanto como primera fonación, etc., ocurren, no sobre un psiquismo virgen, un cero psíquico, sino sobre un psiquismo intrauterino difícil de negar, sea por la más acabada neurociencia, sea por la experiencia en carne propia de cualquier embarazada. El nacimiento, repito, es una ruptura para el neonato de la unicidad con la madre y del confort intrauterino.

Todo lo anterior nos autoriza a plantear sobre el inconsciente cuestiones poco enfocadas en el psicoanálisis clásico: -- a) El mecanismo de clivaje, en su génesis, es anterior a cualquier represión, ya que comienza con el primer traumatismo, -- b) en consecuencia, la huella que dejará en el inconsciente no será una huella mnémica reprimida, -- c) parece pertinente que el psicoanálisis conceda a los fenómenos de clivaje la importancia que nuestros clásicos, con alguna notable excepción, no le dieron.

El autor excepción en aquellos tiempos fue Ferenczi, quien no concede el monopolio a la represión neurótica, sino que se plantea la existencia de fenómenos psíquicos que no caben bajo tal paraguas. Precisión importante: según él, si bien estos mecanismos son claros de observar en las psicosis y las personalidades gravemente traumatizadas, suceden además en el desarrollo de cualquier sujeto. Y se refiere a "impresiones psíquicas, no resueltas ni dominadas, inconscientes, y que nunca posiblemente hayan sido conscientes"1 (post. X). Sobre este inconsciente, confusamente contemplado por Freud, a día de hoy se está produciendo un acuerdo cada vez más amplio en nombrarlo como inconsciente clivado o tramo escindido del inconsciente, previo estructural y genéticamente al reprimido, y que ha venido a cuestionar la teoría y lo que es más importante, la práctica analítica, asunto que abordaré posteriormente. Cito como uno de los autores implicados en este punto de vista a Norberto Marucco (1998).

Si digo que la noción de clivaje en Freud es confusa y aparece y desaparece cual Guadiana, es por datos come el siguiente: en Más allá del principio del placer (1920d), el artículo en que introduce la teoría que habla de pulsiones de vida y de muerte, reflexiona que para que el sueño funcione según el principio del placer, es necesario un trabajo previo. Al que se refiere como una "función... independiente de él [del principio del placer] y que parece más originaria". O también: "habrá que admitir que hubo un tiempo anterior a la tendencia del 
sueño al cumplimiento de deseo". Podemos entender nosotros: que ese tiempo es el del intento de resolución del clivaje.

Y podemos generalizarlo desde el sueño a la actividad psíquica en general: en el psiquismo hay un tiempo anterior y primero al principio del placer. Un tiempo previo de unificación (siempre imperfecta) para, con la ayuda indispensable de un ambiente "vivo" y amoroso, poder tolerar la fragmentación que supuso el "clivaje original". Pero el párrafo de Freud aparece ahí y ahí se queda, porque en buena medida es contradictorio con el resto de sus ideas.

Cabe considerar con él que la angustia esencial, la que modela toda angustia, es la de castración... la esencial que conduce al proceso de represión. Y cabe considerar que hay otro nivel más profundo, más inconsciente por así decir, que no responde a la angustia de castración, ni es defendido mediante la represión, sino mediante el clivaje.

Es un planteo fuerte y que yo mismo me puedo cuestionar, pero como resumen en este punto de lo que en el psicoanálisis tiene o tendría que cambiar para permanecer, diría: El análisis centrado sobre el inconsciente reprimido deja de lado lo escindido, siendo el abordaje de esto segundo la misión fundamental del psicoanálisis actual. El análisis centrado en la represión representa la infancia del psicoanálisis (pongamos el siglo XX). Superada ésta, hemos de añadir y alzaprimar lo escindido.

Escuchando lo anterior, se hace ver que utilizo de forma imprecisa, aunque no indistinta, las nociones de trauma y clivaje. En esencia, como ya comenté antes lo concibo así: el clivaje es la defensa por excelencia del psiquismo ante el trauma. Con lo que nos topamos con otro de los problemas del análisis clásico: el maltrato tanto en la teoría como en la práctica del peso de lo traumatógeno en buena parte de las problemáticas psicopatológicas. Maltrato que comienza en Freud, posiblemente tras descubrir que no podía creer lo que sus "neurotica" le contaban, pues el relato respondía más a fantasías que a sucesos acaecidos. Tal descubrimiento le condujo a abominar de toda teoría traumática, en lo que fue ciegamente seguido por el llamado análisis ortodoxo. Esto condujo a dos clases de problemas, --a) el señalado, del exceso de peso de la fantasía frente a lo proveniente de las vivencias y acciones traumáticas, --b) la infravaloración de lo que sucede con el otro, o mejor dicho (para evitar pensarlo como una noción de Lacan), con los otros significativos, sea lo que sucede benefactor o lesivo.

Dejo aquí el abordaje directo de lo traumático, que sólo cabe expresarlo, como lo he hecho, en una frase, o tendría que dedicarle toda esta conferencia. Me parece más posible reflexionar un poco sobre aquello a lo que he aludido en términos más generales acerca de la relación analítica como vínculo. Dado que en Freud todo era intrapsíquico, tenía escaso 
interés estudiar lo que le sucedía al analizado en la realidad cotidiana, o le había sucedido en épocas pretéritas. Menos aún, hasta bien avanzada su obra, y sólo de forma inconsistente, entendió que el psicoanálisis es una "psicología de dos", como planteará Rickman (citado en Balint 1989 [1970]). Bajo los supuestos de abstinencia e incógnito del analista (alguna vez me enteraré de que quieren decir) la transferencia era considerada como una película presta a desarrollarse en cuanto un psicoanalista se situara en su sillón. No había ningún planteamiento sobre la influencia del analista, y si existía se lo consideraba una indeseable transgresión.

A mi entender esta concepción, aún válida para algunos, ha tenido nefastas consecuencias tanto en la práctica del psicoanálisis, como en su capacidad de impregnación social. En la práctica analítica, desconsiderar la relación analizado-analista, lleva a una frialdad y distancia por parte del terapeuta, a una carencia de todo calor humano, que bien puede transformar al análisis en si mismo en un nuevo traumatismo que viene a incrementar los que traía el paciente. Esta deshumanización además ha conducido al rechazo del psicoanálisis en amplias capas de la sociedad. Esa actitud de los clásicos, de la que forma parte el "flotar como una divinidad por encima del pobre paciente"2 (Ferenczi 1985 [1932]), a la que hay que sumar una reglamentación estricta de cuestiones formales secundarias (la relación debe ser siempre de $\mathrm{Vd}$., el diván es imprescindible, hay que cobrar toda sesión por justificada que esté la ausencia del paciente, lo único que puede hacer el analista es interpretar o mantenerse en silencio, etc.), a esa actitud, repito, es a la que se refiere la consideración del análisis como un lecho de Procusto, personaje mitológico poseedor de una posada, que cuando alojaba a un viajero le adaptaba al tamaño de la cama, bien cortándole las piernas si no cabía, bien descoyuntándole si ocupaba demasiado poco sitio. El psicoanálisis no puede tener ni una brizna de lecho de Procusto.

Se deduce de lo anterior: considero todo análisis como "relacional", aunque debo advertir que lo digo en el sentido de la palabra en el diccionario, no como denominación de origen de una escuela cuya enseñanza no domino. Como dije, este tema del "viejo y nuevo psicoanálisis" anda por mi cabeza desde hace tiempo. Un artículo que escribí hace años lo titulé, "El psicoanalista en el mundo contemporáneo es otro" (2011), queriendo significar que es otro en un doble sentido: es otro en el sentido de distinto al del siglo XX, y otro, porque olvidando neutralidades imposibles, debe funcionar como un "auténtico otro", nada de cómo una ausencia o un espejo o otros símiles de este orden, sino como una presencia que comparte la experiencia analítica del analizado, que se compromete con el paciente.

Judith Dupont (2015), en un reciente libro, critica la ortodoxa actitud de "esperar" al paciente y, muy por el contrario, plantea que el analista debe de salir a la "busca de su paciente", a 
propiciar el encuentro con él. Es una posición grávida en consecuencias: se acabaron los diseños formales rígidos para buscar el que más posible sea para el analizado, se acabaron las teorías monolíticas en las que hay que encajar la personalidad del analizado, y se acabó la pretendida neutralidad (indiferencia, decía Freud).

He comenzado ya con el anterior apartado a pasar de la teoría a cuestiones de tipo técnico, o por mejor decir, cuestiones de práctica analítica, sobre todo porque si se toma el término técnica en sentido estricto, puedo defraudar: no existe una técnica que pueda considerarse como universal. Decía Pontalis que la tarea de cada psicoanalista es inventar el psicoanálisis. Yo iría más lejos: tiene que inventarlo... con cada paciente. Una precisión urgente: habría que salir de la megalomanía de pensar que uno es tan flexible como para poder "encontrarse" con cualquier persona que acuda a su consulta. Habrá vínculos en los que sea imposible establecer una buena "ecuación personal" y que conviene derivarlos hacia otro terapeuta, o quizás a otro tipo de abordaje distinto al psicoanalítico.

Y puesto que tengo ahora la práctica en el foco y hablando de megalomanías, tras situar bajo ese rótulo la creencia en el "psicoanalista universal", voy a hablar de una segunda y no menor: la práctica psicoanalítica no es un "vale todo". A un colega practicante de la psicoterapia, le oí la siguiente afirmación, según él citando a Henry Ey: "Cuanto menos te formes, menos te deformas". Pienso más bien lo contrario. Tomo de Paula Heimann (1950) su comentario acerca de que el psicoanalista Juan o Pedro debe aportar algo más que el hecho de ser Juan o Pedro. Es decir: hay que formarse y prepararse como profesional del psicoanálisis.

He tocado así el tema de la formación en análisis, otro charco que hay que salvar. Para empezar, hablemos de formación de "aprendices de psicoanálisis", o incluso de alumnos, y desterremos el término "candidatos", sólo adecuado para una oposición, o para el candidato a entrar en una secta (los "meritorios" del Opus Dei). Pues así nombrado, considero que lo fundamental es el análisis personal en profundidad del aprendiz. Profundidad que en nada favorece que el analista y quien va a otorgar la potestas, los galones, sean o la misma persona, o bien un colega de la propia institución, con lo que el aprendiz está o puede estar más preocupado de quedar bien que de profundizar en si mismo. Un análisis con alguien que no pertenezca a la misma institución de la que quiere formar parte, se ve mejor facilitador de un análisis "acabado" (decir "terminado" es demasiado decir).

Este requisito de análisis personal me parece el fundamental. También es importante supervisar, cuando menos los primeros tratamientos. Y creo que la formación teórica, aun siendo comparativamente menor, también tiene mucha importancia. Judith Dupont piensa incluso que podría hacerse al margen del análisis personal, bien antes, bien al tiempo, bien expresa. Este material es para uso científico y profesional exclusivamente y puede contener información clínica sensible. Los editores no se responsabilizan de los contenidos de los autores. Dirigir las consultas sobre derechos y autorizaciones a ceir@psicoterapiarelacional.es 
después del mismo. El requisito fundamental es, repito, el análisis personal, del que habría que desterrar las "entrevistas de admisión" (o la passe de los grupos lacanianos), y sólo requerir un determinado número de horas de análisis. Si creemos en nosotros mismos hay que suponer, que si el aprendiz no es adecuado para la profesión de analista, pueda verlo en su análisis.

En resumen; en el dilema entre auctoritas y potestas, se ve inevitable en la formación exigir una potestas, una legalidad para ejercer como psicoanalista, pero el foco debería ponerse más, mucho más, en la auctoritas, la autoridad que deriva del buen hacer del analista.

Otorgado la primacía formativa al análisis personal (llámesele o no didáctico), no es para devaluar la formación mediante seminarios; es más, creo que la formación debe de ser continua, con cualquier edad y con cualquier grado de experiencia. $Y$ ha de ser variada para no caer en el monolitismo teórico, que sobre todo se da cuando el profesional por fuerza se ve obligado a seguir la teoría y la práctica según determinada escuela, pues no conoce otra. Muy al contrario, es necesario tener en la cabeza distintas teorizaciones porque hay distintos analizados, aunque en el momento del encuentro analítico no trate de recordar teorías. Incluso diría, no debe pensar en teorías en el momento de la sesión. Así como Freud hablaba de la "atención flotante" del analista, Piera Aulagnier (1980) piensa en la "teorización flotante", aquella que es evocada por tal o tal analizado. También los seminarios hay que valorarlos, no como lugar de "lecciones magistrales", sino como lugar de intercambio entre los participantes.

Todo lo dicho sobre la formación, recapitulo que lo he hecho pensando en una buena y personalizada práctica. Sobre ella alguna cuestión más.

Me detengo sobre algunos de los principios técnicos presentados por Freud en los años 10 del pasado siglo: de momento por los que prescribe con las metáforas que hablan del analista como un espejo reflectante y como un frío cirujano, y las cuestiones en relación con el manejo de la contratransferencia y con el de la interpretación.

Espejo reflectante y frío cirujano: o sea, ausencia del analista en tanto que persona, y distancia fría hacia el analizado. Ya comenté cómo con un acercamiento a un análisis "relacional", tal actitud resulta inmantenible. ¿Como es eso de ser un espejo reflectante? Pongamos un analista que llena su despacho de adornos que le gustan; pongamos otro que deja las paredes desnudas de toda personalización. ¿Diremos que este segundo, cumpliendo los principios freudianos es el verdaderamente neutral? No está haciendo gala de ser impersonal y distante, ¿qué quiere decir ser neutral? El color negro se define como la ausencia de color, pero es un color muy concreto: el negro. 
Tanto el analista que decora a su gusto el despacho como el que no lo hace, están transmitiendo algo de si mismos. La pretendida neutralidad no existe. Cuando presenté en el pasado este posicionamiento (2014), Franco Borgogno se mostró de acuerdo, pero además profundizaba expresando que ningún analista es neutral. Lo que sucede es que hay analistas que lo reconocen y otros que no.

En cuanto a la contratransferencia, sobre todo en el sentido de lo que despierta en la personalidad del analista, se nos revela hoy, nombrada así o de otras formas, un potente instrumento de conocimiento y de cambio al servicio del analizado. Para Freud nunca pasó del todo de ser una trasgresión. De hecho, el concepto es utilizado por primera vez para nombrar la trasgresión de Jung al convertir a una de sus pacientes en alumna y amante, todo ello a la vez. Y lo que le propone al suizo es "dominarla" por el procedimiento de "endurecer la piel" (Freud / Jung 07/06/1909, en Kerr 93).

Ferenczi, Michel y Alice Balint, Winnicott y Paula Heimann son los fundamentales primeros autores que la conciben de una forma distinta: como un valioso elemento de resonancia del terapeuta con el mundo afectivo del paciente. Quizás gramaticalmente contratransferencia sea un término no del todo adecuado por dos razones: --1) porque parece que siempre es la respuesta a la transferencia del paciente y no considera que en realidad nos estamos refiriendo a una transferencia recíproca, en la que es intento vano delimitar si comienza en analizado o en analista. $Y$--2) Porque en un análisis que no olvide que se trata de dos personas más allá de los lugares que ocupan, no sólo existe lo transferencial /contratransferencial en el análisis, sino toda la riqueza y conflictualidad de un vínculo entre dos personas. Vínculo, además, que algunos pensamos como factor terapéutico importante, e incluso primordial, en la cura analítica. Volveré brevemente sobre ello, pero de momento me centro en una de las consecuencias casi inevitables de tal planteamiento que realza lo vincular como factor de curación:

No está claro a día de hoy que la interpretación (de lo reprimido) sea el momento cumbre y mutativo en el proceso analítico. Expresiones como "la culminación de esta metodología [la del psicoanálisis] se alcanza en el acto interpretativo", o "con la interpretación la función del analista alcanza su punto álgido", citando a Coderch (1995), están muy en cuestión, incluso tal vez para el mismo autor en su evolución. Este cetro concedido a la interpretación es inevitable si se sigue el análisis solipsista de Freud o Klein, pero ya algún coetáneo suyo alertaba contra el "fanatismo de la interpretación" (Ferenczi 1928 III).

Si se sigue el modelo relacional en sentido amplio que vengo de describir, que toma en cuenta la participación e implicación de la persona del analista, la interpretación (según y como) no puede ser descartada, porque adecuadamente utilizada puede abrir nuevos 
campos a explorar, y ayudar a encontrar los nudos realmente conflictivos. Pero cada vez está más lejos de ser considerada como el factor terapéutico por excelencia. Si el marco propuesto por el analista, incluyendo el trabajo interpretativo, es el de una relación cuidadosa, cálida, tolerante, no excesivamente asimétrica, dispuesta a reconocer sus errores, humilde, etc., tal vez sea la relación en si misma el factor curativo por excelencia.

Matizaba que la interpretación "según y como" es válida como forma de exploración y apertura, aunque dejemos de lado su consideración como el culmen de nada. Pero también con "según y como" aludo, espero que coherentemente, a lo que propuse respecto a considerar diferentes tramos del inconsciente, reprimidos unos, clivados o escindidos otros, siendo a estos segundos a los que acuerdo mayor importancia. Al trabajar sobre el inconsciente clivado, si se hace uso de la interpretación, esta tiene que estar guiada en contenido y forma muy diferentes a la "clásica", que tenía por objetivo levantar la barrera de la represión creada por conflictos pulsionales. Y puesto que en la parte clivada no son las angustias derivadas del Edipo y la castración las que están en juego, su utilización como "arte de la sospecha", según la expresión de Paul Ricœur (1999), resulta por completo inadecuada. Si la hostilidad en la transferencia no es hostilidad hacia el rival edípico, sino hostilidad hacia los objetos intrusivos, "trasplantados", depositados en el sujeto y causantes del clivaje traumático, la interpretación tendente a mostrar la rivalidad edípica, sería más que un error, pues no haría sino redoblar el trauma que el analizado trata de superar. El trabajo del analista consiste entonces en ayudar a detectar el (los) objeto(s) importante(s) que actuaron como agentes disruptivos de clivaje por su acción traumatógena.

Ya próximo a terminar. resumo la conferencia llevando al paroxismo la subjetividad de mis criterios: El psicoanálisis para continuar siendo una de las formas de abordaje del sufrimiento psíquico, quizás la más importante, como lo fue en el siglo XX, puede mantener en el actual su esencia, pero no "el tarro de las esencias" ni la "ortodoxia", fea palabra pues hace referencia a la "doxología", ciencia (sic) que estudia las formas correctas e incorrectas de nombrar a la divinidad. Si algunas cuestiones de las que he expuesto como merecedoras de desaparecer o en las que al menos es cuestionable su conveniencia, si aquellas que necesitan ser incorporadas no lo son, estamos asistiendo al punto final de aquel saber que tan útil se reveló hasta ahora.

Aunque quizás todo lo que he expuesto sea un error, lo que por cierto hay que estar dispuesto a admitir con humidad. Cuestiones estas de aceptar los errores y de ser humilde, también obligadas para el psicoanalista en su condición de tal. expresa. Este material es para uso científico y profesional exclusivamente y puede contener información clínica sensible. Los editores no se responsabilizan de los contenidos de los autores. Dirigir las consultas sobre derechos y autorizaciones a ceir@psicoterapiarelacional.es 
Paradójicamente, muchas de las ideas que he expuesto como actuales y futuras, proceden del siglo pasado, del primer tercio del siglo pasado. De Sándor Ferenczi, psicoanalista húngaro cuya dedicación al psicoanálisis dura aproximadamente veinticinco años. Hasta que fallece en 1933. Un alto grado de impregnación de su práctica y su obra han atravesado mi intervención de principio a fin.

Las ediciones en alemán y español de su Diario Clínico de 1932, llevan en portada y bien realzado, el antetítulo "Sin simpatía no hay curación" (1997). Lamentablemente, descontextualizada así, la frase no tiene otro valor que buscar un gancho comercial. Contextualizado, este antetítulo corresponde en realidad a una línea en una de las 200 notas que escribió en 1932, último de su producción. Dicho a vuelapluma, no nos alejamos demasiado si sustituimos una palabra en "Sin simpatía no hay curación", por algo así como "sin vínculo no hay curación", o "sin compromiso no hay curación", o incluso "sin empatía no hay curación".

La anotación que escribe Ferenczi en agosto de 1932, dice,

$$
\text { "Sólo la simpatía cura" (Healing). }
$$

La compresión es necesaria para poder utilizar la simpatía en el lugar adecuado (análisis) y de la manera adecuada. Sin simpatía, no hay curación. (A lo sumo intelecciones sobre la génesis del sufrimiento)". ${ }^{3}$

\section{REFERENCIAS}

Aulagnier, P. El sentido perdido. Buenos Aires: Treb, 1980.

Balint, M. La falta básica. Aspectos terapéuticos de la regresión. Buenos Aires: Paidos, (1989 [1970]).

Coderch, J. La interpretación en psicoanálisis. Barcelona: Herder, 1995.

Dupont, J. Au fil du temps... Paris: Campagne Première, 2015.

Ferenczi, S. Psicoanálisis IV. Madrid: Espasa Calpe. S.A.,1984.

- "Elasticidad de la técnica psicoanalítica" (1928 III)

- "Reflexiones sobre el traumatismo" (post X)

-Journal clinique. Payot. Paris. 1985 (1932); [Sin simpatia no hay curación. El diarío clinico de 1932. Buenos Aires: Amorrortu, 1997]; [Diario Clínico. Buenos Aires: Conjetural, 1988]

Freud, S. Obras completas. Buenos Aires: Amorrortu editores, 1976.

-La interpretación de los sueños (1900a [1899]) expresa. Este material es para uso científico y profesional exclusivamente y puede contener información clínica sensible. Los editores no se responsabilizan de los contenidos de los autores. Dirigir las consultas sobre derechos y autorizaciones a ceir@psicoterapiarelacional.es 
-"Inhibición, síntoma y angustia"(1926d).

-"Proyecto de psicología" (1950 [1895])

- Mas allá del principio del placer (1920g)

-"La escisión del yo en el proceso defensivo" (1940e)

Heinmann, P. "On countertransference". Inter. J. Psycho-anal. 1950. 21, 1-2.

Jiménez Avello, José. --"El psicoanalista en el mundo contemporáneo es otro". En Boschan, Pedro J. (compilador). Sándor Ferenczi y el psicoanálisis del siglo XXI. Buenos Aires: Letra Viva, 2011.

-"Les erreurs fécondes de Ferenczi". Revue Le Coq-Héron, 223. Présence de Ferenczi. Toulouse: Érès, 2015. [Presentación en Journées Internationales du Coq-Héron. Paris, 8 y 9 Marzo 2014].

Kerr. J. A most dangerous method. Nueva York: Alfred A. Knopk, 1993.

Lacan, J. Libro 2, El yo en la teoría del Freud y en la técnica psicoanalítica (1954-1955). Buenos Aires: Paidos, 1983.

Marucco, N.C. Cura analítica y transferencia. Buenos Aires: Amorrortu, 1998.

Rank, Otto. El trauma del nacimiento. Buenos Aires: Paidos (1977 [1924]).

Ricoeur, Paul. Freud: una interpretación de la cultura. México: Siglo Veintiuno, 1999.

Winnicott, D. W. Lettres vives. Paris: Gallimard, 1989. [El gesto espontaneo: Cartas escogidas. Barcelona: Paidos Ibérica, 1990].

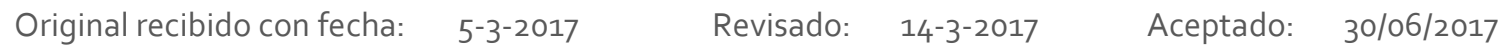

NOTAS:

${ }^{1}$ 26.03.31. "Sobre la revisión de la interpretación de los sueños"

2 01.05.32. ¿Quién está loco, nosotros o los pacientes? (¿Los niños o los adultos?).

3 13.88.32. Registro de los pecados del psicoanálisis 